\title{
Retinol-binding protein 4 is associated with impaired glucose regulation and microalbuminuria in a Chinese population
}

\author{
M. Xu • X. Y. Li • J. G. Wang • X. J. Wang • Y. Huang • \\ Q. Cheng • H. E. Huang • R. Li • J. Xiang • J. R. Tan • \\ M. Dai • G. Ning
}

Received: 22 January 2009 /Accepted: 15 April 2009 /Published online: 9 June 2009

(C) Springer-Verlag 2009

\begin{abstract}
Aims/hypothesis Increased retinol-binding protein 4 (RBP4) has been reported in association with insulin resistance and type 2 diabetes. We aimed to investigate the association of serum RBP4 with impaired glucose regulation and microalbuminuria in Chinese adults aged 40 years or older. Methods Serum RBP4 was measured in 763 individuals with normal glucose regulation, 508 with impaired glucose
\end{abstract}

M. Xu and X. Y. Li contributed equally to this study.

M. Xu • X. Y. Li $\cdot$ X. J. Wang $\cdot$ Y. Huang $\cdot$ J. Xiang $\cdot$ J. R. Tan • M. Dai $\cdot$ G. Ning

Shanghai Clinical Center for Endocrine and Metabolic Diseases, Shanghai Institute of Endocrinology and Metabolism, Rui-Jin

Hospital, Shanghai Jiao-Tong University School of Medicine,

Shanghai, China

J. G. Wang $\cdot$ Q. Cheng

Centre for Epidemiological Studies and Clinical Trials, Rui-Jin

Hospital, Shanghai Jiao-Tong University School of Medicine,

Shanghai, China

H. E. Huang

Centre for Disease Control and Prevention of the Baoshan

District,

Shanghai, China

R. Li

Centre for Disease Control and Prevention of Shanghai,

Shanghai, China

G. Ning $(\bowtie)$

Division of Endocrinology and Metabolism,

The E-Institute of Shanghai Universities, Rui-Jin Hospital,

Shanghai Jiao-Tong University School of Medicine,

Rui-Jin 2nd Road,

Shanghai 200025, China

e-mail: gning@sibs.ac.cn regulation and 524 newly diagnosed diabetic patients. Serum RBP4 was measured using ELISA and urine albumin/ creatinine ratio was used to determine the urinary albumin excretion.

Results Serum RBP4 concentrations were significantly higher in participants with isolated impaired fasting glucose, isolated impaired glucose tolerance, combined impaired fasting glucose/impaired glucose tolerance and diabetes than in those with normal glucose regulation, whereas serum RBP4 levels were not different in the four groups with dysregulation of glucose metabolism. RBP4 was associated with a higher risk for impaired glucose regulation (OR 1.011 for each $1 \mu \mathrm{g} / \mathrm{ml}$ increase in RBP4, 95\% CI 1.000-1.022, $p=0.04$ ) after adjustment for sex, age, BMI, current smoking and alcohol intake, family history of diabetes, insulin resistance, triacylglycerol, total cholesterol, and HDLand LDL-cholesterol; the corresponding OR of combined impaired glucose regulation and type 2 diabetes was 1.022 (95\% CI 1.009-1.035, $p=0.0009)$. RBP4 was associated with the risk of microalbuminuria (OR $1.023,95 \%$ CI 1.004 $1.042, p=0.01$ ) after adjustment for sex, age, smoking habit and alcohol intake, BMI, waist/hip ratio, homeostasis model assessment of insulin resistance, GFR, triacylglycerol, total cholesterol, and HDL- and LDL-cholesterol.

Conclusions/interpretation Serum RBP4 level is closely associated with impaired glucose regulation and is an independent risk factor for microalbuminuria.

Keywords Impaired glucose regulation .

Microalbuminuria $\cdot$ Retinol-binding protein 4

\section{Abbreviations \\ ACR Albumin/creatinine ratio \\ HOMA-IR Homeostasis model assessment of insulin resistance}


IFG Isolated impaired fasting glucose

IGR Impaired glucose regulation

IGT Isolated impaired glucose tolerance

MDRD Modification of diet in renal disease

NGR Normal glucose regulation

RBP4 Retinol-binding protein 4

\section{Introduction}

Recently, retinol-binding protein 4 (RBP4), which is known as a specific transporter that delivers retinol from the liver to other tissues, has been identified as having an effect on systemic insulin sensitivity and glucose homeostasis $[1,2]$. The association of serum RBP4 with BMI and insulin resistance has been most commonly studied; however, the results from these studies are not consistent [3-8]. While serum RBP4 levels were increased in certain metabolic disorders, such as obesity, type 2 diabetes and polycystic ovarian syndrome, that were closely related to insulin resistance $[1-3,9]$, some other studies reported that serum RBP4 levels were not elevated in obese elderly women and that RBP4 level was not related to insulin sensitivity $[5,6]$.

Insulin resistance is a conspicuous characteristic of prediabetic states (impaired fasting glucose [IFG] and/or impaired glucose tolerance [IGT]), plays a crucial role in the development of type 2 diabetes and even contributes to an increased risk of cardiovascular diseases and early mortality $[10,11]$. Elevated serum RBP4 levels have been reported in connection with IGT in studies with small sample sizes [2, 12, 13]; however, the association of RBP4 with isolated IFG, isolated IGT and combined IFG and IGT has rarely been studied. Microalbuminuria, which is an early indicator of renal dysfunction, represents a risk factor for atherosclerosis and cardiovascular disease independent of conventional risk factors $[14,15]$. Previous studies demonstrated that microalbuminuria was associated with insulin resistance in type 2 diabetic patients as well as in non-diabetic individuals $[16,17]$. Studies have also shown an association of serum RBP4 with albumin excretion, although this relationship was not fully elucidated $[2,3]$. In the present study, we investigated the association of serum RBP4 with states of glucose metabolism and urinary albumin excretion in a group of Han Chinese aged 40 years or older.

\section{Methods}

Participants We performed a two-step blood glucose survey in 2004-2005 in a population selected from a single urban community of Shanghai. The study protocol was approved by the Institutional Review Board of the Rui-Jin Hospital and informed consent was obtained from each participant. The study population, design and protocols of this cohort study have been described previously $[18,19]$. Briefly, we first invited all registered permanent residents aged 40 years or older by poster advertisement and by mail to participate in a screening examination, during which we collected information on lifestyle, medical history and the use of medications using a questionnaire, and performed anthropometrical measurements and fasting capillary glucose tests. The smoking habit was defined as 'never', 'current' ( $>6$ months on a daily basis) and 'previous' (cessation of smoking $>6$ months), while the alcohol intake habit was recorded as 'yes' or 'no'. In the second step, after exclusion of individuals with self-reported diabetes mellitus, we classified the screened participants into three groups according to capillary glucose concentrations: $7.0 \mathrm{mmol} / \mathrm{l}$ or above; $5.6-6.9 \mathrm{mmol} / 1$; or below $5.6 \mathrm{mmol} / \mathrm{l}$. We selected randomly a sex- and age-matched sample from these three subgroups for further investigation, which included a $75 \mathrm{~g}$ oral glucose tolerance test and blood and urine sampling. Our selection was based on a ratio of 1:1.2:1.44, as we were concerned that individuals with lower glucose levels might be less likely to participate than those with higher glucose concentrations.

A total of 9,219 individuals $(27 \%$ of the registered permanent residents) participated in the first step of our survey. After the exclusion of 973 individuals with selfreported diabetes, 2,297 (631 people with fasting capillary glucose levels of $7.0 \mathrm{mmol} / \mathrm{l}$ or above; 757 people with levels 5.6-6.9 mmol/1; and 909 individuals with levels below $5.6 \mathrm{mmol} / \mathrm{l}$ ) were invited for further investigation. Eventually 1,835 (491 individuals with fasting capillary glucose level of $7.0 \mathrm{mmol} / \mathrm{l}$ or above; 594 people with levels at 5.6-6.9 mmol/l; and 750 individuals with levels below $5.6 \mathrm{mmol} / \mathrm{l}$ ) participated in the second step of the survey, with an overall retention rate of $80 \%$. The participants $(1,835$ people) and non-participants $(7,384$ people) were similar in characteristics such as sex, age, systolic and diastolic blood pressure. Of 1,835 individuals, 40 were excluded from the present analysis because of missing information: 34 because of incomplete data on fasting, 2 h OGTT, plasma glucose and urinary albumin excretion; and six because of incomplete data on serum RBP4. Thus, 1,795 individuals were involved in the present study, including 763 people with normal glucose regulation, 508 people with impaired glucose regulation and 524 newly diagnosed diabetic patients.

Data collection Body height, body weight, and waist and hip circumferences were measured by the same physician. Body mass index was calculated as body weight in kilograms divided by height squared in metres. Blood pressure was measured at the right arm with an automated electronic device (OMRON Model1 Plus; Omron Company, Kyoto, 
Japan) three times consecutively with 1 min intervals after at least $5 \mathrm{~min}$ rest in the seated position; the three readings were averaged for analysis. Overnight fasting and $2 \mathrm{~h}$ OGTT blood samples were collected in tubes containing EDTA and were centrifuged at $4^{\circ} \mathrm{C}$ and stored at $-80^{\circ} \mathrm{C}$ until analysis. We measured fasting and $2 \mathrm{~h}$ post-load plasma glucose concentrations and serum concentrations of triacylglycerol, total cholesterol, HDL- and LDL-cholesterol, uric acid and creatinine using an autoanalyser (Beckman CX-7 Biochemical Autoanalyser, Brea, CA, USA). We measured serum insulin concentrations using a radioimmunoassay (Sangon Company, Shanghai, China).

Urinary albumin and creatinine concentrations were determined using the first-void sterile urine sample in the early morning by rate nephelometry (Beckman Coulter, Fullerton, CA, USA) and alkaline nitroxanthic acid method, respectively. The sensitivity of the urinary albumin assay was $2.0 \mathrm{~g} / \mathrm{l}$. The albumin/creatinine ratio (ACR) was calculated as milligrams of urinary albumin excretion per gram of urinary creatinine and used for the diagnosis of normal, micro- and macroalbuminuria, defined as an ACR <3.4, 3.4-34, and $>34 \mathrm{mg} / \mathrm{mmol}$, respectively.

ELISA for RBP4 Serum RBP4 concentrations were measured in duplicate by a sandwich ELISA developed in house, using polyclonal and monoclonal antibodies generated against recombinant human RBP4 protein and purified using affinity chromatography [20]. The assay system was subsequently cross-validated by western blotting [20]. The intra-assay $\mathrm{CV}$ was $4.8-11.6 \%$ and inter-assay $\mathrm{CV}$ was $6.3-16.3 \%$ in the present study. Briefly, monoclonal antiRBP4 antibody was used to coat a 96 well microplate overnight at $4^{\circ} \mathrm{C}$. Blocking was performed with $250 \mu \mathrm{l}$ per well PBS containing $1 \%$ (wt/vol.) BSA at $37^{\circ} \mathrm{C}$ for $1 \mathrm{~h}$. After washing three times with PBS containing $0.05 \%$ (vol./vol.) tween-20 (PBST), $100 \mu$ samples of plasma at a dilution of 1:1,000 with PBST containing $0.1 \%$ (wt/vol.) BSA, or purified His-tagged full-length RBP4 $(0.001-0.5 \mu \mathrm{g} / \mathrm{ml})$ as standard, were added and incubated at $37^{\circ} \mathrm{C}$ for $1 \mathrm{~h}$. The plates were washed three times with PBST and then incubated at $37^{\circ} \mathrm{C}$ for $1 \mathrm{~h}$ with $100 \mu \mathrm{l}$ polyclonal antiRBP4 antibody. After washing three times with PBST, the plates were incubated at $37^{\circ} \mathrm{C}$ for $1 \mathrm{~h}$ with $100 \mu \mathrm{l}$ per well detection antibody (horseradish-peroxidase-conjugated antirabbit IgG, Bio-Rad Laboratories, Hercules, CA, USA). The plates were washed three times with PBST, followed by incubation at $25^{\circ} \mathrm{C}$ for $20 \mathrm{~min}$ with $100 \mu$ freshly mixed (1:1) stabilised peroxide solution and chromogen solution (R\&D Systems, Minneapolis, MN, USA). For detection, optical densities were measured at $450 \mathrm{~nm}$ after the reaction was stopped by addition of $100 \mu \mathrm{l} 1 \mathrm{mmol} / 1 \mathrm{H}_{2} \mathrm{SO}_{4}$ per well.

Definitions Impaired glucose regulation was defined as impaired fasting glucose (fasting plasma glucose level $\geq 6.1$ and $<7.0 \mathrm{mmol} / \mathrm{l}$ ) and/or impaired glucose tolerance (IGT, $2 \mathrm{~h}$ OGTT plasma glucose level $\geq 7.8$ and $<11.1 \mathrm{mmol} / \mathrm{l}$ ). Isolated IFG: fasting plasma glucose $\geq 6.1 \mathrm{mmol} / 1$ and $<7.0 \mathrm{mmol} / \mathrm{l}$ and $2 \mathrm{~h}$ OGTT plasma glucose $<7.8 \mathrm{mmol} / \mathrm{l}$. Isolated IGT: $2 \mathrm{~h}$ OGTT plasma glucose $\geq 7.8 \mathrm{mmol} / \mathrm{l}$ and $<11.1 \mathrm{mmol} / \mathrm{l}$ and fasting glucose $<6.1 \mathrm{mmol} / \mathrm{l}$. IFG/IGT: fasting plasma glucose $6.1 \mathrm{mmol} / \mathrm{l}$ to $6.9 \mathrm{mmol} / \mathrm{l}$ and $2 \mathrm{~h}$ OGTT plasma glucose $7.8 \mathrm{mmol} / 1$ to $11.0 \mathrm{mmol} / \mathrm{l}$. Type 2 diabetes was diagnosed according to the 1999 World Health Organization criteria (fasting plasma glucose level $\geq 7.0 \mathrm{mmol} / \mathrm{l}$ and/or $2 \mathrm{~h}$ OGTT plasma glucose level $\geq 11.1 \mathrm{mmol} / \mathrm{l}$ ). A fasting glucose level lower than $6.1 \mathrm{mmol} / \mathrm{l}$ and a $2 \mathrm{~h}$ OGTT plasma glucose level below $7.8 \mathrm{mmol} / \mathrm{l}$ were defined as normal glucose regulation (NGR).

Statistical analysis SAS version 8.1 (SAS Institute, Cary, NC, USA) was used for database management and statistical analysis. The homeostasis model assessment of insulin resistance (HOMA-IR) was calculated according to the equation described by Matthews et al. [21]. The abbreviated Modification of Diet in Renal Disease (MDRD) Study Group formula was used to estimate the glomerular filtration rate based on age, sex, and serum creatinine: GFR $=186.3 \times$ $(0.0133 \times \text { serum creatinine }[\mathrm{mmol} / 1])^{-1.154} \times(\text { age })^{-0.203} \times$ (0.742 if a woman) $\mathrm{ml} \times \min ^{-1} 1.73 \mathrm{~m}^{2}$. Measurements with a skewed distribution were normalised by logarithmic transformation. Comparisons of means and proportions were performed with the standard normal $z$ and $\chi^{2}$ tests, respectively. Homogeneity of groups was determined when the means showed significant differences. Means of these groups were compared by the Student-Newman-Keuls method.

To allow for covariates and confounders, we performed analysis of covariance and multiple linear and logistic regressions. Univariate and multivariable stepwise logistic regression analysis were used to investigate the association of serum RBP4 with clinical and biochemical characteristics. The forward regression procedure was used to obtain determinants of serum RBP4, and we considered as potential covariates age, sex, body mass index, waist circumference, fasting plasma glucose, $2 \mathrm{~h}$ OGTT plasma glucose, $\log _{10}$ insulin, $\log _{10}$ HOMA-IR index, serum concentrations of triacylglycerols, total cholesterol, HDL- and LDLcholesterol, systolic and diastolic blood pressure and $\log _{10}$ ACR. To study the association of impaired glucose regulation with RBP4, we defined participants with normal glucose regulation as $0(n=763)$ and impaired glucose regulation as 1 $(n=508)$, and excluded type 2 diabetic patients from the logistic regression analyses. For the association of combined impaired glucose regulation and type 2 diabetes with RBP4, we defined participants with normal glucose regulation as $0(n=763)$ and combined impaired glucose regulation and type 2 diabetes as $1(n=1,032)$ in the logistic regression 
analyses. To investigate the association of microalbuminuria with RBP4, we defined participants with normal urinary albumin excretion as $0(n=1,651)$ and microalbuminuria as 1 $(n=127)$, excluding macroalbuminuria $(n=17)$ from the logistic regression analyses. For association of combined micro- and macroalbuminuria with RBP4, we defined participants with normal urinary albumin excretion as $0(n=1,651)$ and combined micro- and macroalbuminuria as $1(n=144)$. A $p$ value of less than 0.05 was considered to be statistically significant.

\section{Results}

Characteristics of the participants The study involved 763 participants with NGR, 508 with IGR and 524 with newly diagnosed type 2 diabetes (Table 1). Among the participants with IGR, 116 (6.5\%) had isolated IFG, 252 (14.0\%) had isolated IGT, and 140 (7.8\%) had combined IFG/IGT. There was no significant difference in sex distribution, serum concentration of creatinine and alcohol intake between these groups (all $p>0.05$ ). The average age was 62.5 years and BMI was $25.2 \mathrm{~kg} / \mathrm{m}^{2}$. The NGR and diabetes groups had, respectively, the most favourable and unfavourable metabolic profiles. There was no significant difference in waist circumference, waist/hip ratio, and serum triacylglycerol concentrations between the groups with isolated IFG, isolated IGT and combined IFG/IGT. The isolated IGT and combined IFG/IGT groups had higher serum fasting insulin concentration and urinary ACR than the isolated IFG and NGR groups. The metabolic profile of the combined IFG/IGT group showed a trend towards similarity to the type 2 diabetes group.

Serum RBP4 levels by sex and age Serum RBP4 levels were significantly higher in men than in women $(19.6 \pm 9.8$ vs $17.6 \pm 8.9 \mu \mathrm{g} / \mathrm{ml}, p<0.0001)$. However, with an adjustment for age, waist circumference, waist/hip ratio, current smoking, current alcohol intake and education, the difference became non-significant $(p=0.57)$. Age was positively associated with serum RBP4 levels $(r=0.07, p=0.005)$. $\mathrm{RBP} 4$ concentrations increased by $0.60 \mu \mathrm{g} / \mathrm{ml}$ with each 10 year increase in age $(p=0.01)$.

Determinants of serum RBP4 concentrations Serum triacylglycerol, total cholesterol, HDL- and LDL-cholesterol, waist/hip ratio, sex, and $\log _{10}$-transformed urinary ACR were independent determinants for serum RBP4 concentrations in a stepwise linear regression analysis (Table 2). However, RBP4 levels were not associated with either serum C-reactive protein $(r=0.04, p=0.13)$ or white blood cell count $(r=0.05, p=0.71)$.
RBP4 in impaired glucose regulation and newly diagnosed type 2 diabetes In both sexes combined, serum RBP4 concentrations were significantly increased in participants with isolated IFG, isolated IGT, combined IFG and IGT, and newly diagnosed type 2 diabetes compared with concentrations in participants with NGR (19.1, 19.8, 19.2, $19.6 \mu \mathrm{g} / \mathrm{ml}$ vs $16.8 \mu \mathrm{g} / \mathrm{ml}$, all $p<0.01$ ), after adjustment for sex, age, BMI, current smoking and current alcohol intake, the education received and the family history of diabetes. There was no statistical difference between these four groups with dysregulation of glucose metabolism (all $p>0.51$ ) (Fig. 1).

As shown in Table 3, increased RBP4 was associated with an increased risk of hyperglycaemia. The higher level of RBP4 had a higher risk of impaired glucose regulation (OR 1.011, 95\% CI 1.000-1.022, $p=0.04$ ) after adjustment for sex, age, BMI, current smoking and alcohol intake, family history of diabetes, insulin resistance, triacylglycerol, total cholesterol, HDL- and LDL-cholesterol; the corresponding OR for both impaired glucose regulation and type 2 diabetes was 1.022 (95\% CI 1.009-1.035, $p=0.0009)$.

Serum RBP4 and the risk of albuminuria We also found that serum RBP4 concentrations were increased from $18.1 \mu \mathrm{g} / \mathrm{ml}$ in participants with normal albumin excretion, to $21.1 \mu \mathrm{g} / \mathrm{ml}$ in those with microalbuminuria, and to $25.2 \mu \mathrm{g} / \mathrm{ml}$ in those with macroalbuminuria $(p<0.0001)$; and the difference remained statistically significant after adjustment for sex, age, BMI, waist circumference, waist/ hip ratio, HOMA-IR, current smoking and alcohol intake, serum triacylglycerol, plasma glucose, blood pressure and use of antihypertensive drugs; there was no significant difference between the micro- and macroalbuminuria groups ( $p=0.82$; Fig. 2). Increased serum RBP4 was associated with high risk of microalbuminuria and combined micro- and macroalbuminuria (Table 4). The logistic regression analysis also showed that a $1 \mu \mathrm{g} / \mathrm{ml}$ increase in RBP4 level was associated with an increased risk of microalbuminuria and combined micro- and macroalbuminuria of $2.3 \%(p=0.01)$ and $2.4 \%(p=0.006)$, respectively, even after adjustment for sex, age, smoking habit and alcohol intake, BMI, waist/hip ratio, HOMA-IR, glomerular filtration rate, triacylglycerol, total cholesterol, and HDLand LDL-cholesterol (Table 4).

\section{Discussion}

In the present study we found that, in a large Chinese cohort aged 40 years or older, serum RBP4 concentrations were higher in the presence of isolated IFG, isolated IGT, combined IFG and IGT, and type 2 diabetes. Serum RBP4 


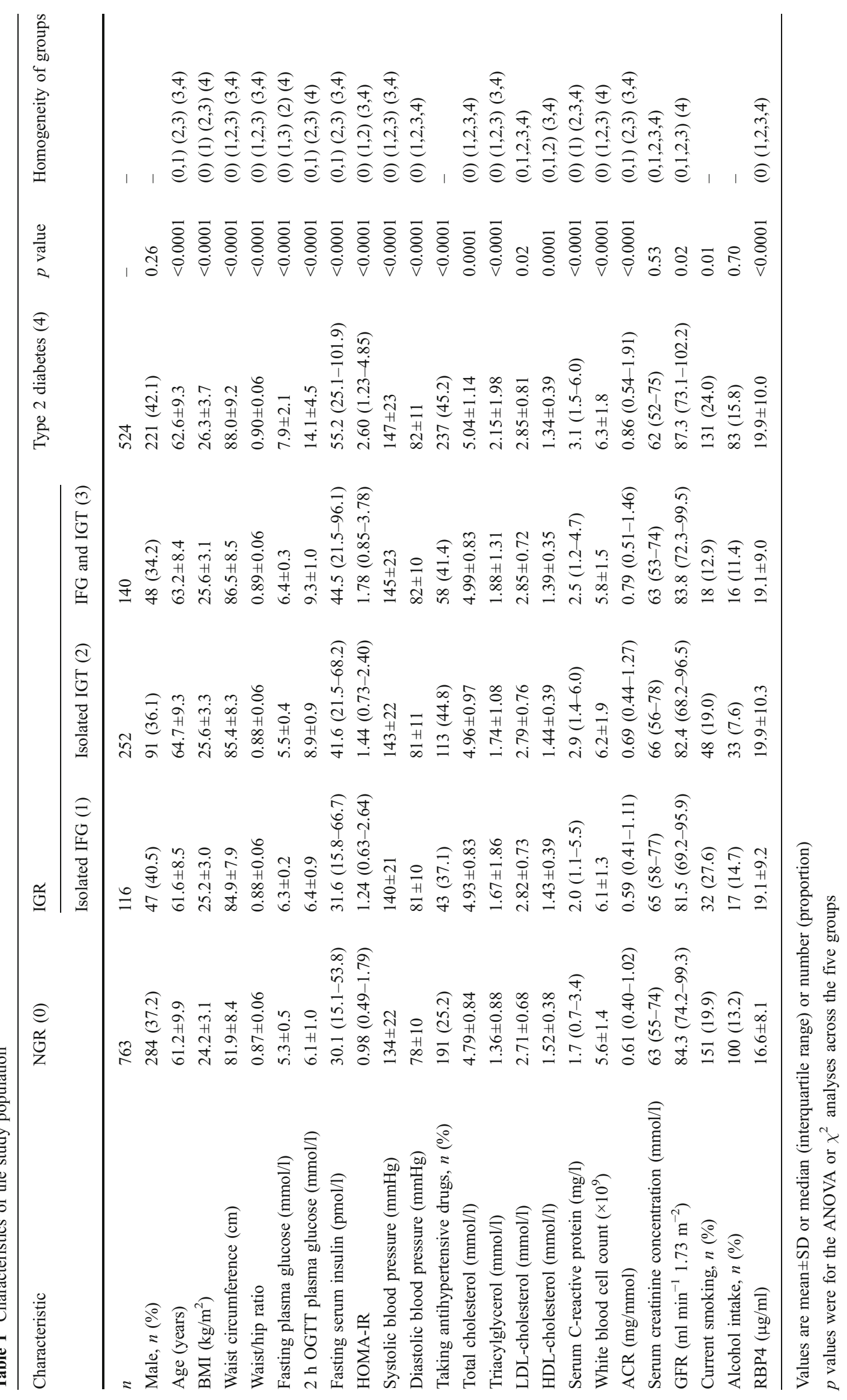


Table 2 Univariate and stepwise regression analysis with serum RBP4 concentration as a dependent variable

\begin{tabular}{|c|c|c|c|c|c|}
\hline \multirow[t]{2}{*}{ Co-variable } & \multicolumn{3}{|c|}{ Univariate } & \multicolumn{2}{|l|}{ Stepwise } \\
\hline & $r$ & \pm SEM & $p$ value & \pm SEM & $p$ value \\
\hline Age (years) & 0.07 & $0.06 \pm 0.03$ & 0.02 & - & - \\
\hline Sex $($ male $=1$, female $=2)$ & -0.10 & $-2.04 \pm 0.50$ & $<0.0001$ & $-1.85 \pm 0.47$ & $<0.0001$ \\
\hline Body mass index $\left(\mathrm{kg} / \mathrm{m}^{2}\right)$ & 0.09 & $0.16 \pm 0.13$ & 0.21 & - & - \\
\hline Waist circumference $(\mathrm{cm})$ & 0.15 & $0.13 \pm 0.06$ & 0.05 & - & - \\
\hline Waist/hip ratio & 0.20 & $29.10 \pm 5.90$ & $<0.0001$ & $22.88 \pm 3.80$ & $<0.0001$ \\
\hline Fasting plasma glucose (mmol/l) & 0.11 & $1.01 \pm 0.51$ & 0.05 & - & - \\
\hline 2 h OGTT plasma glucose $(\mathrm{mmol} / \mathrm{l})$ & 0.13 & $0.08 \pm 0.08$ & 0.30 & - & - \\
\hline $\log _{10}$ fasting serum insulin $(\mathrm{pmol} / \mathrm{ml})$ & 0.10 & $15.1 \pm 9.0$ & 0.09 & - & - \\
\hline $\log _{10}$ HOMA-IR & 0.12 & $16.1 \pm 8.9$ & 0.07 & - & - \\
\hline Triacylglycerol (mmol/l) & 0.21 & $2.51 \pm 0.31$ & $<0.0001$ & $2.59 \pm 0.30$ & $<0.0001$ \\
\hline Total cholesterol (mmol/l) & 0.13 & $-2.30 \pm 0.61$ & 0.0002 & $-2.36 \pm 0.60$ & $<0.0001$ \\
\hline HDL-cholesterol (mmol/l) & 0.004 & $5.03 \pm 0.73$ & $<0.0001$ & $4.86 \pm 0.73$ & $<0.0001$ \\
\hline LDL-cholesterol (mmol/l) & 0.07 & $3.45 \pm 0.69$ & $<0.0001$ & $3.51 \pm 0.68$ & $<0.0001$ \\
\hline Systolic blood pressure (mmHg) & 0.08 & $0.03 \pm 0.01$ & 0.07 & - & - \\
\hline Diastolic blood pressure (mmHg) & 0.10 & $0.06 \pm 0.03$ & 0.03 & - & - \\
\hline $\log _{10}$ ACR & 0.09 & $0.98 \pm 0.52$ & 0.05 & $1.14 \pm 0.50$ & 0.02 \\
\hline
\end{tabular}

$\beta$, Regression coefficient; $r$, Pearson correlation coefficient

was also associated with a higher risk of microalbuminuria independent of various confounding factors. However, given the cross-sectional nature of our study, no causal inference can be drawn.

Serum RBP4 levels were positively correlated with several metabolic indices, such as BMI, waist/hip ratio, HOMA-IR, blood pressure, impaired glucose metabolism (IFG/IGT, type 2 diabetes) and serum triacylglycerol. These findings were in line with previous studies [2, 3]. We did

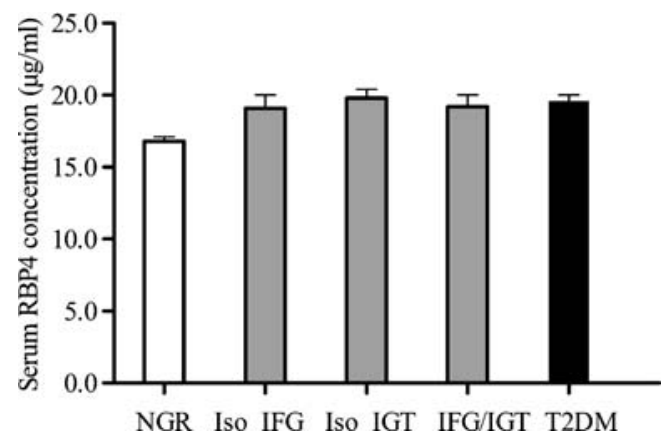

Fig. 1 Adjusted means $( \pm$ SEM) of serum RBP4 concentrations in participants with NGR, IGR (isolated IFG [Iso IFG], isolated IGT [Iso IGT] and IFG/IGT) and type 2 diabetes mellitus (T2DM). The covariables included age, sex, BMI, current smoking, current alcohol intake, the education received and the family history of diabetes. Participants with type 2 diabetes mellitus and IGR (isolated IFG, isolated IGT and IFG/IGT) had higher concentrations of RBP4 than those with NGR (both $p<0.0001$ ). There was no statistical difference among the subgroups of IGR $(p=0.67)$ and between the IGR and type 2 diabetes mellitus groups $(p=0.71)$ not find a positive correlation between serum RBP4 and BMI or waist circumference in a multivariate linear regression analysis. Nevertheless, we found waist/hip ratio to be one of the independent determinants of the serum RBP4 concentration. Compared with the wide range of BMI in the report by Graham et al. $\left(23.0-40.9 \mathrm{~kg} / \mathrm{m}^{2}\right)$ [2], the BMI range was relatively narrow in this study. Consequently, this may contribute to the non-significant difference in serum RBP4 levels between obese and nonobese individuals. However, we assumed that our findings provided the evidence that RBP4 was highly correlated with obesity, especially central obesity.

In our study, we found the waist/hip ratio to be independently and positively associated with RBP4. The INTERHEART study investigators argued that the prevalence of obesity was underestimated when based on BMI values. If a raised waist/hip ratio was used to assess the risk of cardiovascular disease, the proportion of people classified as obese worldwide would increase substantially, especially in the Middle East, southern Asia and SouthEast Asia [22]. Thus, the waist/hip ratio might be a more accurate index of obesity in the present population. Also, some recent studies [4-6] failed to detect difference in circulating RBP4 levels according to obese status. Janke et al. [6] argued that adipose tissue might be a less important source of circulating RBP4 in humans than in animals and that it was possible that the increase in circulating RBP4 in the insulin-resistant state was not explained by increased RBP4 production in adipose tissue. Thus, prospective and 
Table 3 The risk of impaired glucose regulation and type 2 diabetes associated with a $1 \mu \mathrm{g} / \mathrm{ml}$ increase in serum RBP4

\begin{tabular}{|c|c|c|c|c|c|}
\hline Model & Adjustment & IGR $(n=508)$ & $p$ value $^{\mathrm{a}}$ & $\begin{array}{l}\text { Impaired glucose regulation } \\
\text { and type } 2 \text { diabetes } \\
(n=1,032)\end{array}$ & $p$ value $^{\mathrm{b}}$ \\
\hline Model 1 & Unadjusted & $1.038(1.024-1.052)$ & $<0.0001$ & $1.040(1.028-1.052)$ & $<0.0001$ \\
\hline Model 2 & $\begin{array}{l}\text { Adjusted for age, sex, current smoking, current } \\
\text { alcohol intake and family history of diabetes }\end{array}$ & $1.038(1.024-1.052)$ & $<0.0001$ & $1.040(1.028-1.052)$ & $<0.0001$ \\
\hline Model 3 & $\begin{array}{l}\text { Further adjusted for BMI and waist/hip ratio, } \\
\text { based on Model } 2\end{array}$ & $1.034(1.020-1.049)$ & $<0.0001$ & $1.033(1.021-1.046)$ & $<0.0001$ \\
\hline Model 4 & Further adjusted for HOMA-IR, based on Model 3 & $1.032(1.018-1.042)$ & $<0.0001$ & $1.029(1.017-1.042)$ & $<0.0001$ \\
\hline Model 5 & $\begin{array}{l}\text { Further adjusted for serum triacylglycerol, total } \\
\text { cholesterol, HDL- and LDL-cholesterol, based on } \\
\text { Model } 4\end{array}$ & $1.011(1.000-1.022)$ & 0.04 & $1.022(1.009-1.035)$ & 0.0009 \\
\hline
\end{tabular}

Values are ORs $(95 \% \mathrm{CI})$

${ }^{a}$ For the risk of IGR, we defined participants with normal glucose regulation as $0(n=763)$ and IGR as $1(n=508)$, excluding type 2 diabetic patients $(n=524)$ from the analysis

${ }^{\mathrm{b}}$ For the risk of combined IGR and type 2 diabetes, we defined participants with NGR as $0(n=763)$ and combined IGR and type 2 diabetes as 1 $(n=1,032)$

biological studies are needed to further explore the association of serum RBP4 with obesity.

Increased RBP4 levels increased the risk for hyperglycaemia, including impaired glucose regulation and newly diagnosed type 2 diabetes, after excluding the effects of age, sex, central obesity, HOMA-IR, family history of type 2 diabetes and the levels of serum lipid. Yang et al. [1] reported that increasing RBP4 could act directly to induce the expression of phosphoenolpyruvate carboxykinase (PEPCK) (also known as PCK2), increase glucose production and reduce insulin action to suppress glucose production in hepatocytes. In addition, there is a report explaining that RBP4 attenuates insulin-induced phosphorylation of insulin

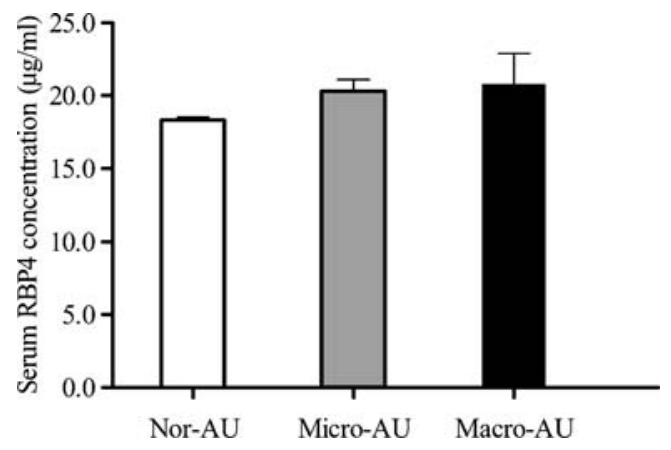

Fig. 2 Adjusted means $( \pm$ SEM) of serum RBP4 concentrations in participants with normal urinary albumin excretion (Nor-AU) $(n=$ $1,651)$, microalbuminuria (Micro-AU) $(n=127)$ and macroalbuminuria (Macro-AU) $(n=17)$. The co-variables included sex, age, BMI, waist circumference, waist/hip ratio, HOMA-IR, current smoking and alcohol intake, serum triacylglycerol, plasma glucose, blood pressure and use of antihypertensive drugs. There was no significant difference between the groups with micro- and macroalbuminuria $(p=0.82)$, while the RBP4 level was significantly higher in the group with microalbuminuria than in the normal group $(p=0.01)$ receptor substrate 1 (IRS1) and extracellular regulated kinase (ERK1/2) in primary human adipocytes [23]. Several studies have shown that after treatment with thiazolidinediones (TZDs), an insulin sensitiser, or exercise, serum RBP4 levels are decreased and insulin resistance improves $[2,12]$.

We found a striking association between serum RBP4 and lipid levels, especially levels of triacylglycerol. Our results were in line with previous findings $[2,3,5]$. Insulin resistance could be the potential mechanism for increased serum lipid levels. However, we found that when we controlled for serum insulin, insulin resistance and waist/ hip ratio, serum RBP4 independently predicted the risk of hypertriacylglycerolaemia. It was suggested that RBP4 might have a direct role in the progression of lipogenesis. In a very recent study [24], RBP4 increased the expression of the gene encoding fatty acid synthase (FASN) in adipose tissue in a manner predominantly correlating with visceral fat accumulation, impaired insulin sensitivity and levels of circulating adipokines.

We also found a positive association of serum RBP4 with albumin excretion and the independent association of RBP4 with risk of microalbuminuria, which coincides with the findings from Takebayashi [3]. The concentrations of RBP4 were significantly elevated in the plasma of diabetic patients and were significantly higher in those with microalbuminuria [25]. Raila et al. report that despite higher excretion of RBP4 in the urine, the levels of RBP4 were still elevated in the plasma of type 2 diabetic patients [25]. In the present study, we found that the prevalence of microalbuminuria increased correspondingly and significantly with the increase in serum RBP4 concentration. RBP4 independently increased the risk of microalbuminuria. Microalbuminuria, which is an early indicator of renal dysfunction, also behaves as a risk factor for arteriosclerosis 
Table 4 The risk of micro- and macroalbuminuria associated with a $1 \mu \mathrm{g} / \mathrm{ml}$ increase in serum RBP4

\begin{tabular}{|c|c|c|c|c|c|}
\hline Model & Adjustment & $\begin{array}{l}\text { Microalbuminuria } \\
(n=127)\end{array}$ & $p$ value $^{\mathrm{a}}$ & $\begin{array}{l}\text { Micro- and macroalbuminuria } \\
(n=144)\end{array}$ & $p$ value $^{\mathrm{b}}$ \\
\hline Model 1 & Unadjusted & $1.038(1.024-1.052)$ & $<0.0001$ & $1.034(1.018-1.050)$ & $<0.0001$ \\
\hline Model 2 & $\begin{array}{l}\text { Adjusted for age, sex, current smoking, current } \\
\text { alcohol intake and family history of diabetes }\end{array}$ & $1.029(1.012-1.046)$ & 0.0008 & $1.033(1.010-1.048)$ & $<0.0001$ \\
\hline Model 3 & $\begin{array}{l}\text { Further adjusted for BMI and waist/hip ratio, } \\
\text { based on Model } 2\end{array}$ & $1.025(1.008-1.043)$ & 0.004 & $1.029(1.013-1.046)$ & 0.0003 \\
\hline Model 4 & Further adjusted for HOMA-IR, based on Model 3 & $1.025(1.008-1.043)$ & 0.004 & $1.029(1.013-1.046)$ & 0.0003 \\
\hline Model 5 & $\begin{array}{l}\text { Further adjusted for glomerular filtration rate, based } \\
\text { on Model } 4\end{array}$ & $1.028(1.010-1.046)$ & 0.002 & $1.031(1.014-1.048)$ & 0.0003 \\
\hline Model 6 & $\begin{array}{l}\text { Further adjusted for serum triacylglycerol, total } \\
\text { cholesterol, HDL- and LDL-cholesterol, based } \\
\text { on Model } 5\end{array}$ & $1.023(1.004-1.042)$ & 0.01 & $1.024(1.007-1.042)$ & 0.006 \\
\hline
\end{tabular}

Values are ORs $(95 \% \mathrm{CI})$

${ }^{\text {a }}$ For the risk of microalbuminuria, we defined participants with the normal urinary albumin excretion as $0(n=1,651)$ and microalbuminuria as 1 $(n=127)$, excluding macroalbuminuria $(n=17)$ from the analysis

${ }^{\mathrm{b}}$ For the risk of combined micro- and macroalbuminuria, we defined participants with normal urinary albumin excretion as $0(n=1,651)$ and combined micro- and macroalbuminuria as $1(n=144)$

and cardiovascular disease independent of conventional risk factors.

Given the findings that RBP4 levels were extensively associated with the indicators of insulin resistance, such as hyperglycaemia and hypertension, RBP4 might play a role in endothelial dysfunction though the mechanism of impaired insulin sensitivity. Microalbuminuria is well established as one of the risk factors for cardiovascular disease, suggesting that serum RBP4 might serve as an alternative biomarker of cardiovascular disease. This needs confirmation from future prospective studies.

We were not able to find a significant relation between RBP4 and inflammation, as indicated by the levels of Creactive protein and white blood cell counts, in our study. Inflammation is thought to suppress hepatic RBP4 mRNA synthesis [26] and decrease plasma concentrations of RBP4 and retinol [27, 28]. The association of RBP4 with inflammation remains controversial. Sell et al. [29] studied the regulation of RBP4 production by adiponectin and TNF- $\alpha$ in primary human adipocytes; they found that TNF$\alpha$ strongly downregulates RBP4 production in adipocytes, a completely unexpected effect as TNF- $\alpha$-treated adipocytes are insulin resistant. The association of RBP4 with inflammation, either acute or low-grade chronic inflammation, is of particular interest.

A major strength of our present study was that none of participants had used glucose-lowering drugs or insulin, so the confounding effect of glucose-lowering treatment could be excluded. Several limitations of our study have also to be addressed. First, although our study participants originated from an epidemiological study, they should not be regarded as a randomly selected sample from the general population, nor as one comprising entirely volunteers, as those who agreed to participate already enrolled in the epidemiological study, which involved $27 \%$ of all eligible inhabitants with permanent residence in the community. Possible selection bias cannot be ruled out. Second, our study included more women than men, because the participation rate was higher among women. In China, women retire from work 5 years earlier than men. Indeed, in our present study, fewer women than men had to go to work. Third, the values of serum RBP4 were relatively lower than those reported by Graham et al. [2], which could reflect the different racial origins of the participants. In several reports on Asian populations, the RBP4 levels were comparably lower than those in the white population for non-diabetic individuals or diabetic patients $[3,12,13]$. The other explanation may be the different measuring system used. Graham et al. [30] recently reported that competitive enzyme-linked immunoassays may selectively underestimate serum RBP4 levels in the setting of insulin resistance because of assay saturation. They also concluded that the quantitative western blotting standardised to full-length RBP4 protein was a 'gold standard' method for measuring serum RBP4 in the insulin-resistant state. An additional reason for the discrepancy may be that the antibodies used in the ELISA had different affinities.

In conclusion, we reported that serum RBP4 levels were closely related to impaired glucose metabolism and other metabolic disorders and were independently associated with a higher risk of microalbuminuria in Chinese adults. Central obesity, determined by waist/hip ratio, and triacylglycerol levels were highly correlated with increased serum RBP4 concentrations. Prospective studies are needed to elucidate the role of serum RBP4 in the pathogenesis of type 2 diabetes and cardiovascular disease. 
Acknowledgements The present study would not have been possible without the participation of the participants. This work was supported by the grants from 863 Project (No. 2006AA02A409), Nature Science Foundation of China (No. 30725037) and E-Institute of Shanghai Universities, Shanghai Education Commission (No. E03007 and No. Y0204).

Duality of interest The authors declared that there is no duality of interest associated with this manuscript.

\section{References}

1. Yang Q, Graham TE, Mody N et al (2005) Serum retinol binding protein 4 contributes to insulin resistance in obesity and type 2 diabetes. Nature 436:356-362

2. Graham TE, Yang Q, Bluher M et al (2006) Retinol-binding protein 4 and insulin resistance in lean, obese, and diabetic subjects. N Engl J Med 354:2552-2563

3. Takebayashi K, Suetsugu M, Wakabayashi S, Aso Y, Inukai T (2007) Retinol binding protein-4 levels and clinical features of type 2 diabetes patients. J Clin Endocrinol Metab 92:2712-2719

4. Broch M, Vendrell J, Ricart W, Richart C, Fernandez-Real JM (2007) Circulating retinol binding protein 4, insulin sensitivity, insulin secretion and insulin disposition index in obese and nonobese subjects. Diabetes Care 30:1802-1806

5. von Eynatten M, Lepper PM, Liu D et al (2007) Retinol-binding protein 4 is associated with components of the metabolic syndrome, but not with insulin resistance, in men with type 2 diabetes or coronary artery disease. Diabetologia 50:1930-1937

6. Janke J, Engeli S, Boschmann M et al (2006) Retinol-binding protein 4 in human obesity. Diabetes 55:2805-2510

7. Gavi S, Stuart LM, Kelly P et al (2007) Retinol-binding protein 4 is associated with insulin resistance and body fat distribution in nonobese subjects without type 2 diabetes. J Clin Endocrinol Metab 92:1886-1890

8. Stefan N, Hennige AM, Staiger H, Schleicher E, Fritsche A, Häring HU (2007) Circulating retinol-binding protein-4, insulin sensitivity, insulin secretion, and insulin disposition index in obese and nonobese subjects: response to Broch et al. Diabetes Care 30:e91

9. Tan BK, Chen J, Lehnert H, Kennedy R, Randeva HS (2007) Raised serum, adipocyte, and adipose tissue retinol-binding protein 4 in overweight women with polycystic ovary syndrome: effects of gonadal and adrenal steroids. J Clin Endocrinol Metab 92:2764-2772

10. Reaven GM (2005) Insulin resistance, the insulin resistance syndrome, and cardiovascular disease. Panminerva Med 47:201-210

11. Cersosimo E, DeFronzo RA (2006) Insulin resistance and endothelial dysfunction: the road map to cardiovascular diseases. Diabetes Metab Res Rev 22:423-436

12. Jia W, Wu H, Bao Y et al (2007) Association of serum retinol binding protein 4 and visceral adiposity in Chinese subjects with and without type 2 diabetes. J Clin Endocrinol Metab 92:3224-3229

13. Cho YM, Youn BS, Lee $\mathrm{H}$ et al (2006) Plasma retinol-binding protein-4 concentrations are elevated in human subjects with impaired glucose tolerance and type 2 diabetes. Diabetes Care 29:2457-2461
14. Stehouwer CDA, Gall MA, Twisk JWR, Knudsen E, Emeis JJ, Parving HH (2002) Increased urinary albumin excretion, endothelial dysfunction, and chronic low-grade inflammation in type 2 diabetes. Diabetes 51:1157-1165

15. Mykkanen L, Zaccaro DJ, O'Leary DH, Howard G, Robbins DC, Haffner SM (1997) Microalbuminuria and carotid artery intimamedia thickness in nondiabetic and NIDDM subjects: the Insulin Resistance Atherosclerosis Study (IRAS). Stroke 28:1710-1716

16. Parvanova AI, Trevisan R, Iliev IP et al (2006) Insulin resistance and microalbuminuria. Diabetes 55:1456-1462

17. Mykkanen L, Zaccaro DJ, Wagenknecht LE, Robbins DC, Gabriel M, Haffner SM (1998) Microalbuminuria is associated with insulin resistance in nondiabetic subjects: the Insulin Resistance Atherosclerosis Study. Diabetes 47:793-800

18. Li XY, Xu M, Wang JG et al (2009) Serum C-reactive protein (CRP) and microalbuminuria in relation to fasting and 2$\mathrm{h}$ postload plasma glucose in a Chinese population. Clin Endocrinol (Oxf) 70:691-697

19. Xiang J, Li XY, Xu M et al (2008) Zinc transporter-8 gene (SLC30A8) is associated with type 2 diabetes in Chinese. J Clin Endocrinol Metab. 93:4107-4112

20. Qi Q, Yu Z, Ye X et al (2007) Elevated retinol-binding protein 4 levels are associated with metabolic syndrome in Chinese people. J Clin Endocrinol Metab 92:4827-4834

21. Matthews RD, Hosker JP, Rudenski AS, Naylor BA, Treacher DF, Turner RC (1985) Homeostasis model assessment: insulin resistance and cell function from fasting plasma glucose and insulin concentrations in man. Diabetologia 28:412-419

22. Yusuf S, Hawken S, Ounpuu S et al (2005) Obesity and the risk of myocardial infarction in 27000 participants from 52 countries: a case-control study. Lancet 366:1640-1649

23. Ost A, Danielsson A, Liden M, Eriksson U, Nystrom FH, Stralfors P (2007) Retinol-binding protein-4 attenuates insulininduced phosphorylation of IRS1 and ERK1/2 in primary human adipocytes. FASEB J 21:3696-3704

24. Berndt J, Kovacs P, Ruschke K et al (2007) Fatty acid synthase gene expression in human adipose tissue: association with obesity and type 2 diabetes. Diabetologia 50:1472-1480

25. Raila J, Henze A, Spranger J, Mohlig M, Pfeiffer AF, Schweigert FJ (2007) Microalbuminuria is a major determinant of elevated plasma retinol-binding protein 4 in type 2 diabetic patients. Kidney Int 72:505-511

26. Rosales FJ, Ritter SJ, Zolfaghari R, Smith JE, Ross AC (1996) Effect of acute inflammation on plasma retinol, retinol-binding protein, and its mRNA in the liver and kidneys of vitamin Adeficiency rats. J Lipid Res 37:962-971

27. Schweigert FJ (2001) Inflammation-induced changes in the nutritional biomarkers serum retinol and carotenoids. Curr Opin Clin Nutr Metab Care 4:477-481

28. Stephensen CB (2001) Vitamin A, infection, and immune function. Annu Rev Nutr 21:167-192

29. Sell H, Eckel J (2007) Regulation of retinol binding protein 4 production in primary human adipocytes by adiponectin, troglitazone and TNF- $\alpha$. Diabetologia 50:2221-2223

30. Graham TE, Wason CJ, Bluher M, Kahn BB (2007) Shortcomings in methodology complicate measurements of serum retinol binding protein (RBP4) in insulin-resistant human subjects. Diabetologia 50:814-823 